Supporting information

\title{
STM Imaging of electron migration in real space and time: a simulation study
}

\author{
YanHo Kwok, ${ }^{1,2}$ GuanHua Chen, ${ }^{1, \text { a) }}$ and Shaul Mukamel ${ }^{3, b)}$
}

${ }^{1}$ Department of Chemistry, the University of Hong Kong, Pofkulam Road, Hong Kong,

${ }^{2}$ QuantumFabless Limited, Sha Tin, Hong Kong,

${ }^{3}$ Department of Chemistry and Physics and Astronomy, University of California, Irvine, California 92617, United States 


\section{Effect of pulse-width and frequency}

The choices of pulse-width and frequency are important to obtain a good agreement between the time-dependent populations and the integrated current signals. Figure S1 shows the excited populations of site 1-6 after the illumination of a single laser pulse with central frequency $\omega$ and full-width at half-maximum $(\mathrm{FWHM})=20$ fs. The peak positions correspond to the HOMOLUMO gaps of the corresponding sites while the peak widths are determined by the pulse-width and the line width due to the electrodes.

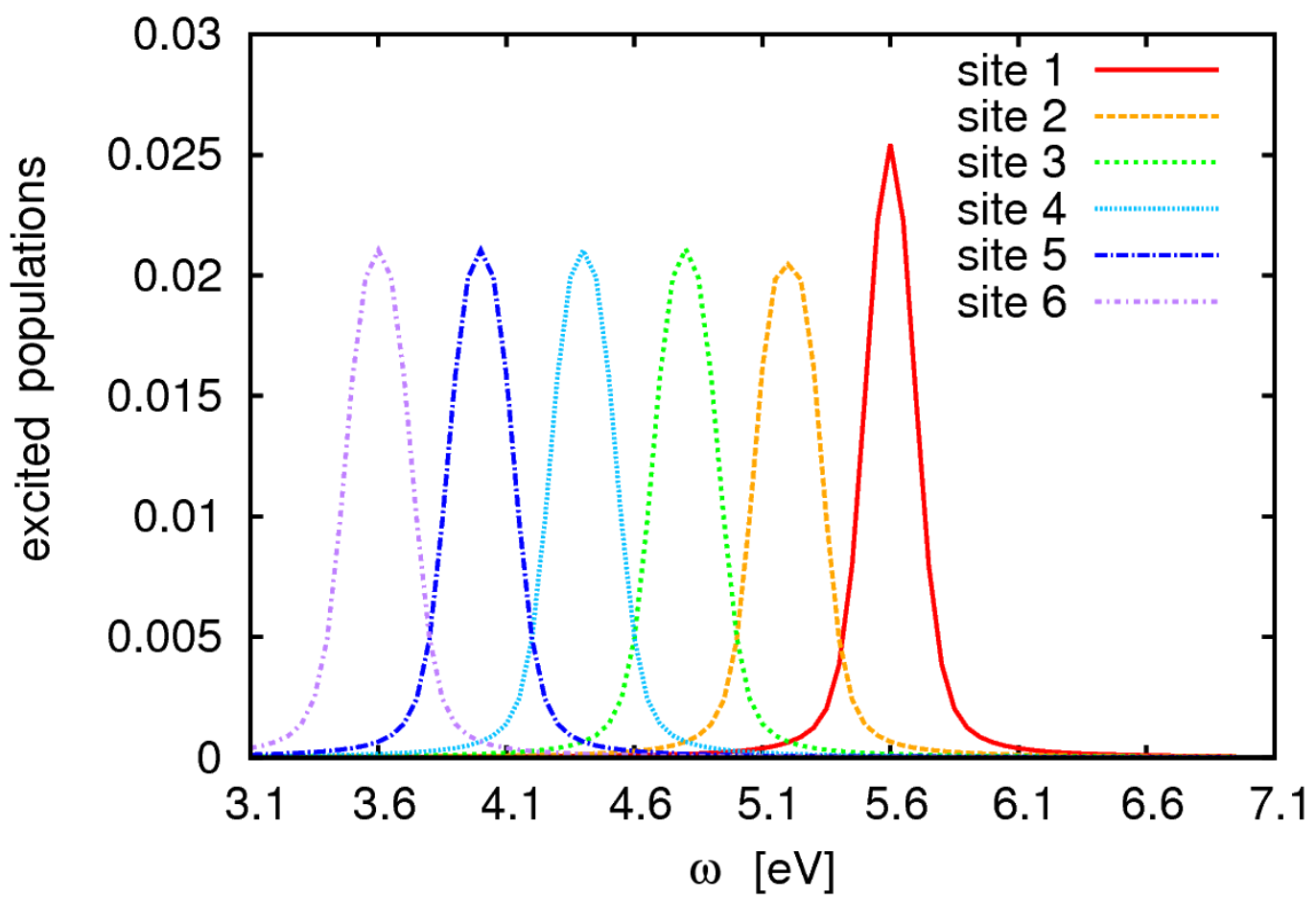

Figure S1. Excited populations on site 1-6 after a single laser pulse with frequency $\omega$ and $\mathrm{FWHM}=20 \mathrm{fs}$. 
The time-resolution of a pump-probe experiment is limited by the width of the pulses. Therefore the pulse-width has to be comparable to or shorter than the timescale of phenomenon of interest. On the other hand, a short pulse also means a wide bandwidth in the energy domain and this will cause unwanted excitation of the other sites even though the frequency of the pulse is not in resonant with them. This unwanted excitation will finally lead to unwanted current signal. Here, we compare simulation results with FWHM being set to 12fs and 20fs (both pump and probe pulse have the same FWHM), which are shown in Figure S2. Other parameters remain the same as given in the main text. We can see that even for FWHM $=12 \mathrm{fs}$, the integrated current curves still follow the time-dependent populations. But we will see that the larger bandwidth does cause more unwanted current due to excitations of nearby sites.

(a)

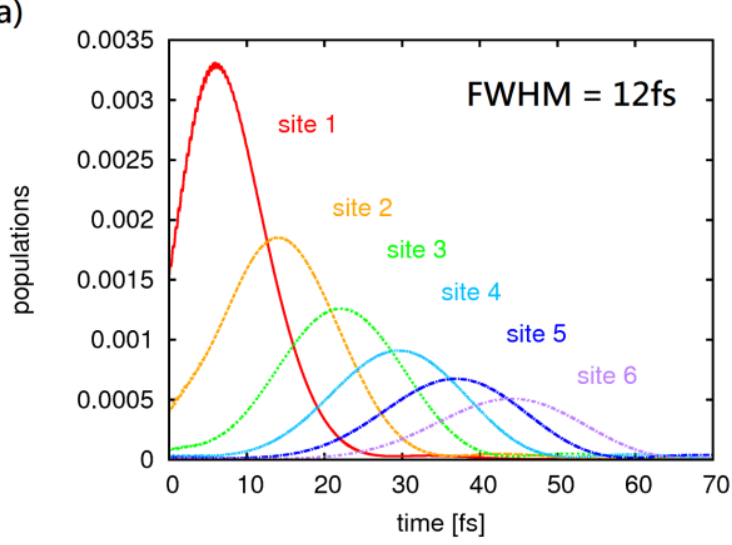

(c)

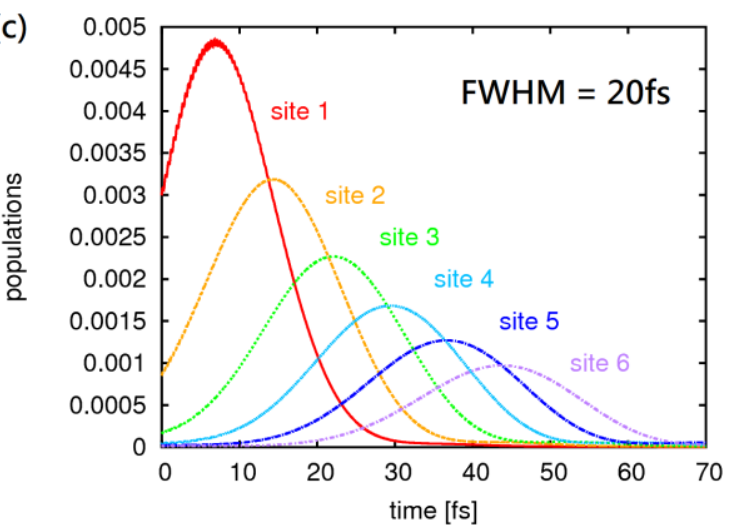

(b)

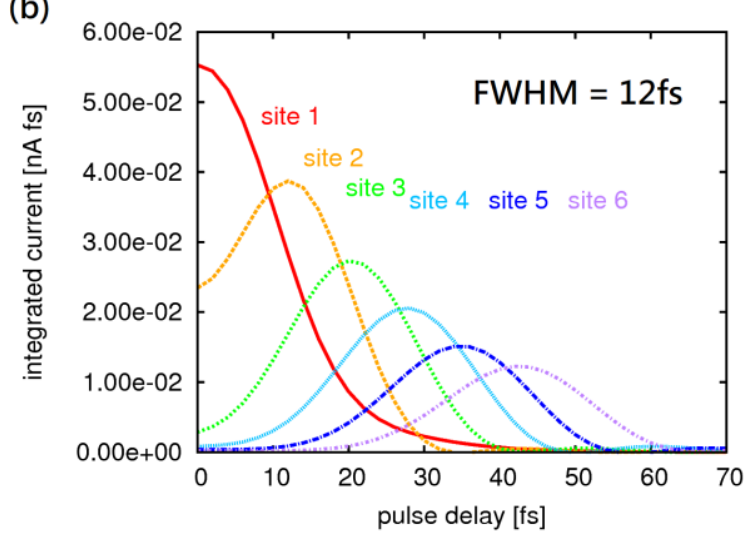

(d)

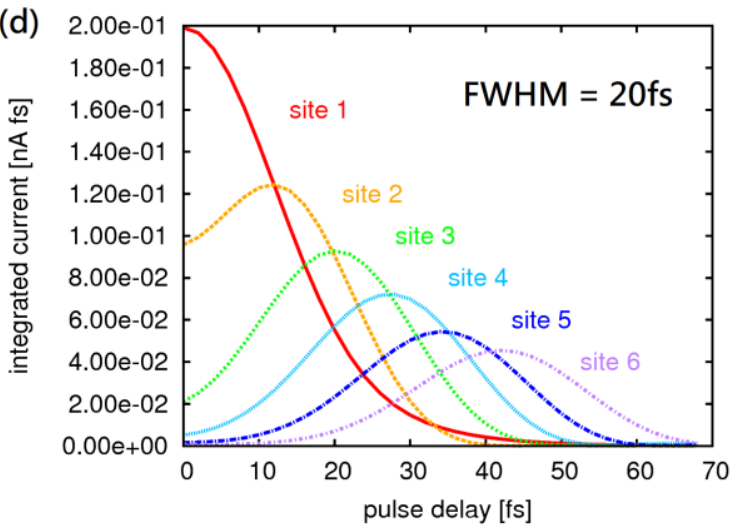


Figure S2. (a) Populations versus real time for sites 1-6 with FWHM = 12fs. (b) Integrated current versus pump-probe pulse delay for tip located at site 1-6 with FWHM $=12 \mathrm{fs}$. For the tip located at site 1 , the magnitude is reduced by 3 times. (c) Populations versus real time for sites 1 6 with FWHM = 20fs. (d) Integrated current versus pump-probe pulse delay for tip located at site 1-6 with FWHM = 20fs. For the tip located at site 1, the magnitude is reduced by 3 times.

The integrated current resulting from unwanted excitation can be estimated by modifying the dipole matrix $\boldsymbol{\mu}$ such that the pump can only excite site 1 while the probe can only excite the site under tip. This would give the current free from unwanted excitation, which is shown as point curves in Figure S3. The original integrated currents are plotted as line curves for reference. The difference between the line curves and point curves corresponds to the unwanted current. The unwanted current is significant and contributes to the total current for a large portion when the pulse delay is short. This is due to the large population of site 1 just after the pump pulse arrives, therefore unwanted excitation of site 1 by the probe will cause a significant additional current. Also, the additional current with FWHM $=12 \mathrm{fs}$ is more significant than that with FWHM $=20 \mathrm{fs}$, which is expected due to the larger energy bandwidth of the pulses.
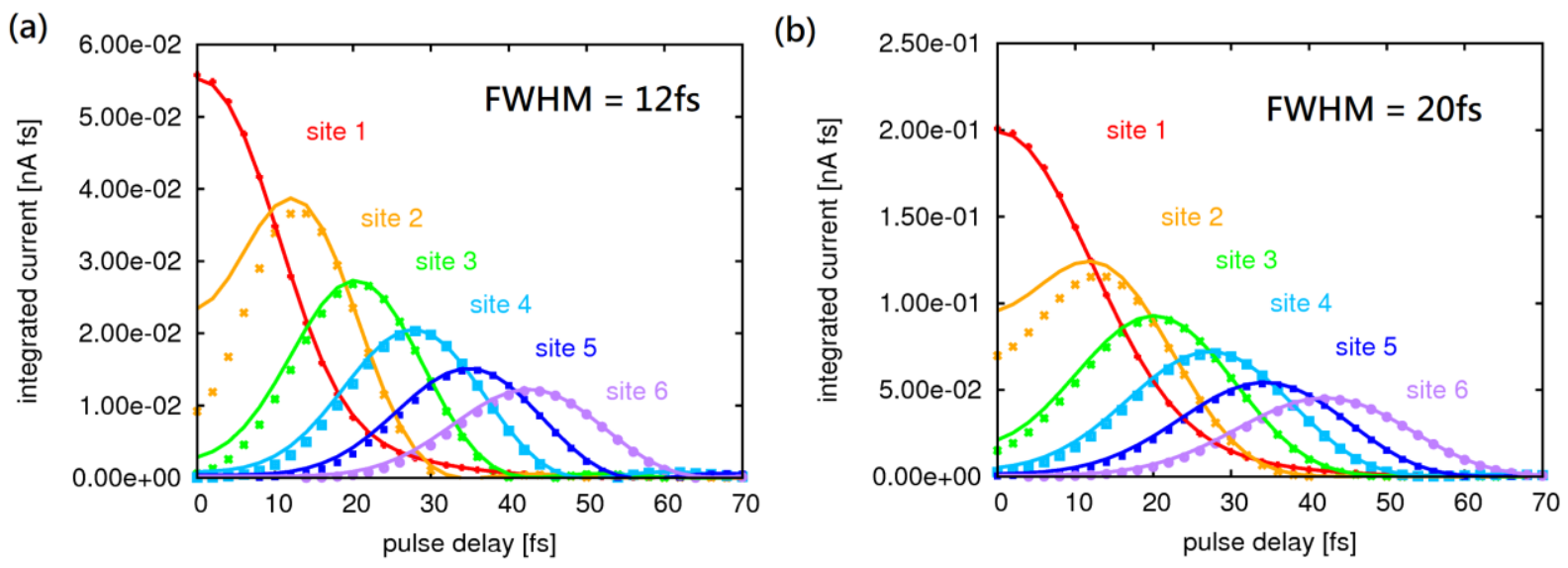
Figure S3. Integrated current versus pulse delay for tip at site 1-6 respectively with (a) FWHM = 12fs and (b) FWHM = 20fs. Solid line curves are integrated current with unwanted excitation. Point curves are integrated current free from unwanted excitation (obtained by modifying dipole matrix so that pump pulse only excites site 1 and probe pulse only excites the tip-probing site). For the tip located at site 1, the magnitude is reduced by 3 times.

\section{Effect of coupling with substrate}

The coupling between the system and the substrate leads to dissipative effect which drives the excited system back to equilibrium gradually. Such effect is quantified by the linewidth matrix. The larger the linewidth, the faster the dissipation occurs and the excited populations decay faster. In the manuscript, the linewidth matrix due to coupling with the substrate is diagonal with value equal to $0.02 \mathrm{eV}$ for the LUMO. This value is comparable to the choice of hopping among sites, which is $0.05 \mathrm{eV}$, and the excited electrons in the LUMO dissipate in the timescale of around tens of femtoseconds. In reality, the molecule may be placed on a thin decoupling layer to avoid strong coupling with the substrate. To simulate that situation, we reduce the linewidth of the LUMO due to the substrate by 10 times, from $0.02 \mathrm{eV}$ to $0.002 \mathrm{eV}$, which would lead to a much slower decay of the populations. All the other parameters are kept the same. Fig. S4(a) shows the time-dependent populations in this case. We can see that the populations decay much slower. Figure S4(b) shows the integrated current versus pulse delay. We can see that the curves still follow with the time-dependent populations. 

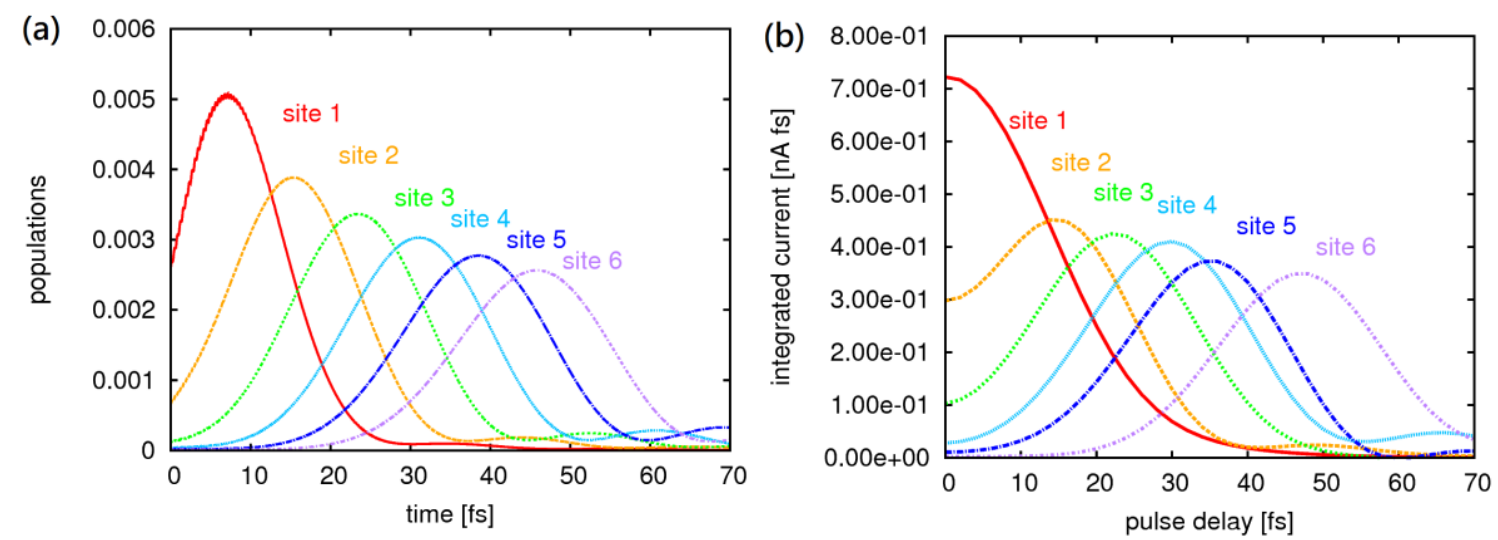

Figure S4. Simulation results with reduced linewidth. (a) LUMO populations of sites 1-6 induced by the pump. (b) Integrated current versus pulse delay for tip at site 1-6 respectively. For the tip located at site 1 , the magnitude is reduced by 3 times.

\section{Charge injection and the effect of asymmetric pulses}

As mentioned in the main content, the laser pulses can affect the system in two ways. First, the laser pulses can excite electrons from HOMO to LUMO when the frequency is resonant with the HOMO-LUMO gap. Second, when the polarization of the electric field is parallel to the tipsubstrate direction, a significant transient bias voltage can be induced due to field enhancement. The induced transient voltage can cause the injection of electrons from the electrodes.

Here, we attempt to estimate the effect of electron injection when the field is polarized along tipsubstrate direction. Let us recall that the incident electric field is of the form

$$
E(t)=\mathcal{E}_{1}(t) \cos \left(\omega_{1} t\right)+\mathcal{E}_{2}(t) \cos \left(\omega_{2} t\right)
$$

We assume that after field-enhancement, a transient voltage of the same form, with peak voltage equals to $2.0 \mathrm{~V}$, is induced across the junction. This value is large but possible in experiments 
considering the fact that the field enhancement factor can be over thousands ${ }^{1,2}$. Since the LUMO of the sites are $1.5 \mathrm{eV}$ above the Fermi level while HOMO are $0.7-2.5 \mathrm{eV}$ below, for temperature at $300 \mathrm{~K}$, this induced voltage in principle allows electron injection into or out of the system.

The actual distribution of the enhanced field depends on the actual system of interest. Here, we still treat the enhanced field by the dipole approximation. And we assume the tip-substrate distance is $10 \AA ̊$ so that the enhanced field would be $0.2 \mathrm{~V} / \AA$. . The transition dipole matrix elements for all sites are assumed to be $0.2 \mathrm{e} \AA$. This value is smaller than that when the field is perpendicular to the tip-substrate direction because we assume the molecule under study is lying approximately flat on the substrate. Other parameters, such as the line width, pulse width and the DC voltage, remain the same as given in the main text. The transient bias voltage $V_{\alpha}(t)$ is taken into account in our equation of motion for the dissipative matrices.

$$
\begin{gathered}
\boldsymbol{\varphi}_{\alpha, p}(t)=-i R_{p} \int_{-\infty}^{t} d \tau \mathbf{G}_{D}^{r}(t, \tau) \boldsymbol{\Gamma}_{\alpha} \exp \left(-i \int_{t}^{\tau} d t^{\prime} \varepsilon_{\alpha, p}+V_{\alpha}\left(t^{\prime}\right)\right) . \\
i \frac{d}{d t} \boldsymbol{\varphi}_{\alpha, p}(t)=-i R_{p} \boldsymbol{\Gamma}_{\alpha}+\left[h_{D}-\frac{i}{2} \boldsymbol{\Gamma}-\varepsilon_{\alpha, p}-V_{\alpha}(t)\right] \boldsymbol{\varphi}_{\alpha, p}(t),
\end{gathered}
$$

Figure S5 shows the results with effect of transient bias taken into account. First of all, figure S5(a) shows the time-dependent populations for the LUMO of sites 1-6 due to the pump. The arrival time of the center of the pump pulse is set to zero. The transient voltage causes electron injection from the electrodes occurs when the pump pulse arrives and contributes to additional populations on the LUMO of the sites. Such additional populations are plotted versus time in figure S5(b). The populations gradually increase during the pump but are highly oscillatory due to the highly oscillating transient voltage which drives the current in and out the system. This approximated cancellation of positive and negative transient currents make the net electrons 
injected small compared to the populations due to resonant excitation. After the pump, the injected populations gradually leave through the electrodes. Figure S5(c) shows the summation of such additional populations again for sites 1-6 but with different phase-shifted pump pulse.

$$
E(t)=\mathcal{E}_{1}(t) \cos \left(\omega_{1} t+\phi\right)
$$

Such phase shift can introduce significant asymmetry for single-cycle $\mathrm{THz}$ pulses ${ }^{2,3}$. But since we are using multi-cycles pulse that last for tens of periods, shifting the phase only has little effect on the symmetry. Thus the results are similar. Figure S5(d) shows the integrated current versus pulse delay for tip at site 1-6. Electron injection due to transient bias also affects the integrated current signal, contributing to additional integrated current for short pulse delay. Nevertheless, the integrated current signals still follow the populations.

Finally, it is remarked that the electric field $(0.2 \mathrm{~V} / \mathrm{A})$ we are considering here is indeed large and it may cause tunneling field emission. We simulate this situation in order to demonstrate that the electron injection would not cause a problem even at such induced transient voltage. In reality, we can of course reduce the incident field strength according to the field enhancement factor to avoid field emission. 
(a)

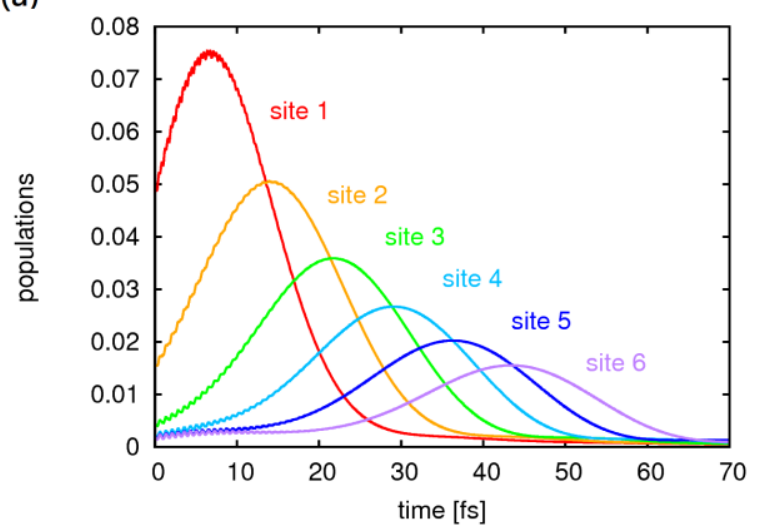

(c)

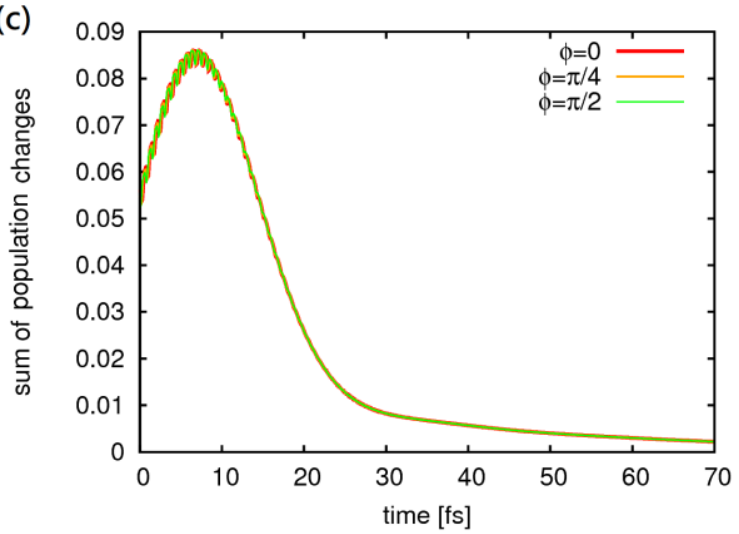

(b)
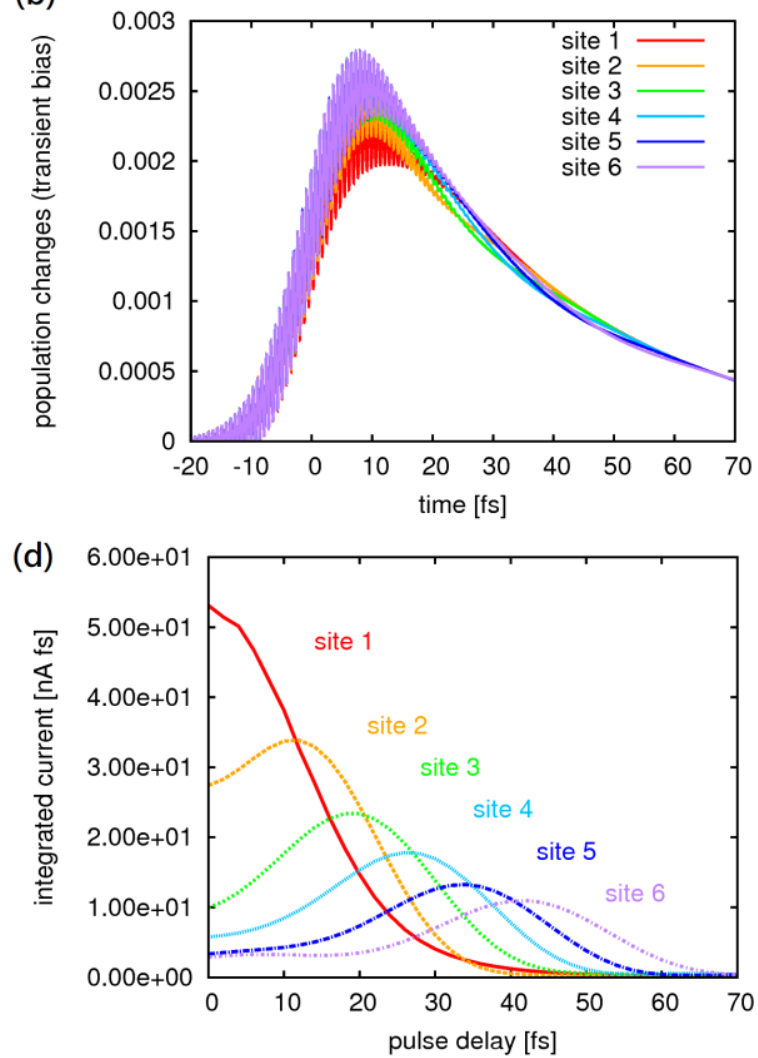

Figure S5. Simulation results with transient bias effect. (a) LUMO occupations of sites 1-6 induced by the pump. (b) LUMO occupation changes of sites 1-6 due to the induced transient voltage (c) summation of the occupation changes for sites 1-6 due to the induced transient voltage for different phase for the pump pulse. (d) Integrated current versus pulse delay for tip at site 1-6 respectively. For the tip located at site 1, the magnitude is reduced by 3 times.

\section{System with varying LUMO energies}

In the model presented in the main content, we assume the same LUMO energies $(1.5 \mathrm{eV}$ above the Fermi level) for all sites for simplicity. Here, we consider a system with gradually decreasing LUMO energies. The LUMO energies of the 10 sites are 1.5, 1.47, 1.44, 1.41, 1.38, 1.35, 1.32, 1.29, 1.26 and $1.23 \mathrm{eV}$ respectively. The difference between LUMO energies of neighboring 
sites is comparable to the hopping $(0.05 \mathrm{eV})$, so the charge migration still occurs smoothly. Again, the central frequency of the pump is $5.6 \mathrm{eV}$ and the central frequency of the probe is chosen to be resonant with the HOMO-LUMO gap of the site under the STM tip. All the other parameters are kept the same. Figure S6(a) shows the time-dependent populations in this case and figure S6(b) shows the integrated current versus pulse delay. Compared to the original model, the time-dependent populations in this case show additional peaks at later time due to the varying LUMO energies. Still, the integrated current curves follow the time-dependent populations.
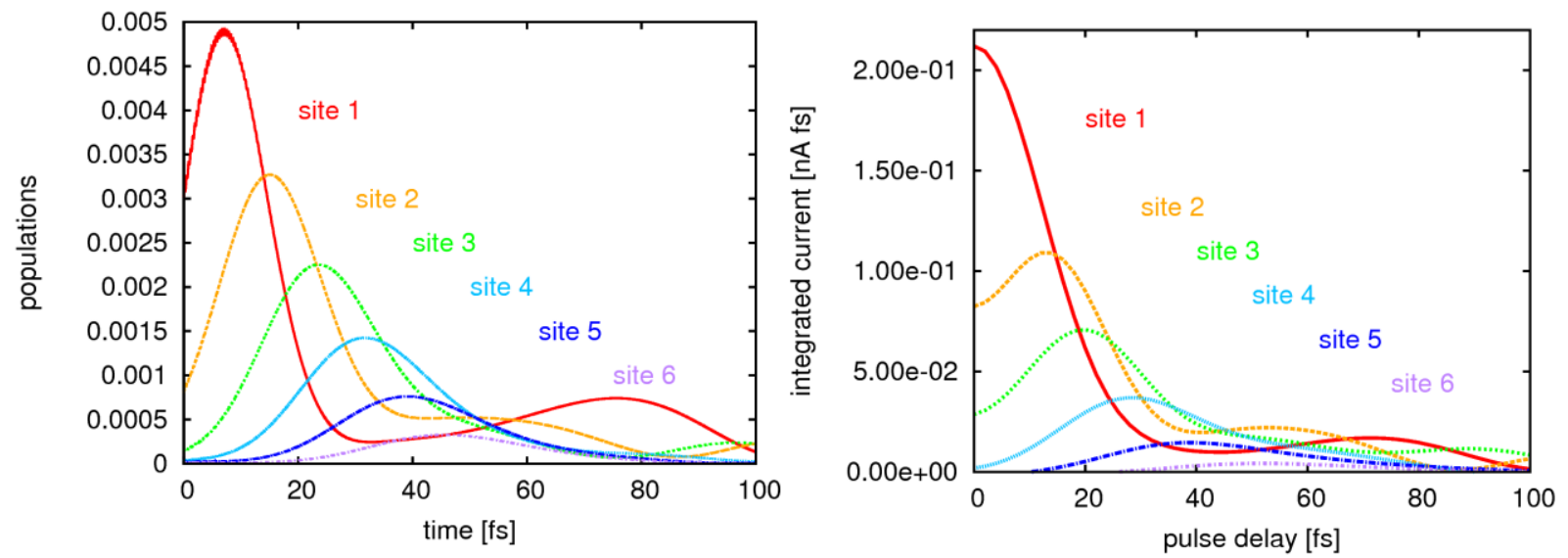

Figure S6. Simulation results with varying LUMO energies. (a) LUMO populations of sites 1-6 induced by the pump. (b) Integrated current versus pulse delay for tip at site 1-6 respectively. The magnitude is reduced by 4 times for tip at site 1 and 1.5 times for tip at site 2 .

\section{References}

(1) Cocker, T.; Jelic, V.; Gupta, M. Nat Photonics 2013, 7, 620-625. 
(2) Yoshioka, K.; Katayama, I.; Minami, Y.; Kitajima, M.; Yoshida, S.; Shigekawa, H.; Takeda, J. Nat. Photonics 2016, 10, 762-765.

(3) Cocker, T. L.; Peller, D.; Yu, P.; Repp, J.; Huber, R. Nature 2016, 539, 263-267. 